\title{
Clinical Complete Response After Neoadjuvant Therapy for Esophageal Cancer: What's Lurking Under the Surface?
}

\author{
Lorenzo Ferri, MD, PhD \\ David Mulder Chair in Surgery, Division of Thoracic Surgery, Upper GI Cancer Program, Montreal General Hospital, \\ McGill University, Montreal, Canada
}

In this edition of the Annals of Surgical Oncology, Drs. Ohkura and colleagues from one of Japan's premier esophageal cancer centers, the Toranomon Hospital in Tokyo run by Harushi Udagawa, describe their experience with the management of clinical complete response after neoadjuvant intent therapy (primarily chemoradiotherapy) of esophageal squamous cell carcinoma. The authors compare outcomes of patients in whom a subsequent esophagectomy was performed to those that had surveillance alone. Selection of treatment, surgical versus surveillance, was primarily driven by patient preference in this series. The authors elected to include only neoadjuvant intent and not patients treated with definitive chemoradiation to reduce bias. Although laudable, this reduces the sample size significantly, possibly affecting power. A profound difference in both disease and recurrence-free survival is reported in this series, with no difference in overall survival. The authors attribute this as possibly due to late pulmonary related deaths in the resection group, although the very small number of patients who died postresection $(n=5)$ limits any meaningful conclusions regarding the cause of death.

The decision to operate on a patient with esophageal cancer who has had a complete clinical response after induction therapy is controversial, and there are limited data to help direct an informed approach. Recently, more clinicians have elected to pursue a "wait and see" approach after neoadjuvant therapy for esophageal cancer with complete clinical response. The reasons for this are

(C) Society of Surgical Oncology 2018

First Received: 22 April 2018;

Published Online: 14 May 2018

L. Ferri, MD, $\mathrm{PhD}$

e-mail: lorenzo.ferri@mcgill.ca understandable. Esophagectomy, particularly in the eyes of many oncologists and the public, is perceived as a high-risk surgery with poor outcomes resulting in a reduced quality of life. Why incur the risk of this procedure, if the cancer is gone?

However, the data supporting this selective surgery approach are not definitively supported in the literature. Although two prior, randomized trials exploring a trimodality versus bimodality (without surgery) approach for esophageal squamous cell carcinoma failed to show a difference in overall survival, both studies were hampered by unacceptable operative mortality rates of approximately $10 \%$ potentially offsetting any oncologic benefit of resection. ${ }^{1,2}$ A one-in-ten mortality rate is inconceivable in this era of centralization of complex oncology surgery into high-volume centers in which mortality can be limited to $1-2 \%$. Indeed, in the present series, from one of Japan's premier esophageal surgery programs, the postoperative mortality rate was zero. Furthermore, both studies showed a significant reduction in local-regional recurrence with surgery, a finding confirmed by the present smaller singleinstitution series from the Toranomon Hospital.

Central to a selective approach to surgery after induction therapy is the accuracy of complete clinical response to represent complete pathological response. If complete clinical response is equivalent to complete pathological response, then a selective approach for surgery is entirely justifiable. The present Toranomon Hospital experience demonstrates a decent rate of concordance between clinical and pathological response, with only $28 \%$ of resected patients having residual malignancy. Unfortunately, this reasonable rate of accuracy is not the case in most previous series on the topic. Complete clinical response implies that all investigations, both invasive and noninvasive, fail to demonstrate active malignant disease after induction treatment. Endoscopic surveillance (EGD and EUS \pm 
FNA) is notoriously inaccurate for detecting residual disease and noninvasive tests (CT and PET) fare no better. ${ }^{3,4}$ What is the downside of waiting until a subclinical residual focus of malignancy becomes clinically apparent? The risk is that a resectable lesion may, in the interval during surveillance, become unresectable, a not inconceivable notion given the limitations of surveillance methods. This is particularly concerning for lesions in the mid or upper thorax with potential for invasion into adjacent great vessels and airway. With these largely ineffective surveillance techniques, is it not surprising that in most series, a large proportion of patients with complete clinical response harbor residual disease? In a mixed histology retrospective series from MD Anderson that reflects most published reports, more than $60 \%$ of patients with complete clinical response undergoing esophagectomy had residual cancer in the resection specimen leading the authors to conclude "Surgery-eligible GEC (gastro-esophageal cancer) patients should be encouraged to undergo surgery following chemoradiation despite achieving a clinical complete response". 5 I agree with this conclusion in patients with an acceptable surgical risk, particularly for mid and proximal esophageal cancers for which in waiting for the malignancy to recur one may miss a curable window.

\section{REFERENCES}

1. Stahl M, Stuschke M, Lehmann N, et al. Chemoradiation with and without surgery in patients with locally advanced squamous cell carcinoma of the esophagus. J Clin Oncol. 2005;23(10):2310-7.

2. Bedenne L, Bouche O, Milan C, et al. Chemoradiation followed by surgery compared with chemoradiation alone in squamous cancer of the esophagus: FFCD 9102. J Clin Oncol. 2007;25(10):1160-8.

3. van Rossum PS, Goense L, Meziani J, et al. Endoscopic biopsy and EUS for the detection of pathologic complete response after neoadjuvant chemoradiotherapy in esophageal cancer: a systematic review and meta-analysis. Gastrointest Endosc. 2016;83(5):866-79.

4. Noordman BJ, Wijnhoven BPL, Lagarde SM, et al. Active surveillance in clinically complete responders after neoadjuvant chemoradiotherapy for esophageal or junctional cancer. Dis Esophagus. 2017;30(12):1-8.

5. Cheedella NK, Suzuki A, Xiao, et al. Association between clinical complete response and pathological complete response after preoperative chemoradiation in patients with gastroesophageal cancer: analysis in a large cohort. Ann Oncol. 2013;24(5):1262-6. 